\title{
A FOCUSING COLLECTOR FOR LONG WAVELENGTH X-RAY ASTRONOMY*
}

\author{
D. J. YENTIS, J. R. P. ANGEL, D. MITCHELL, R. NOVICK, and P. VANDEN BOUT \\ Columbia Astrophysics Laboratory, Columbia University, New York, N.Y., U.S.A.
}

\section{Introduction}

One of the outstanding problems in X-ray astronomy is the detection and understanding of a number of important phenomena that are expected at photon energies in the range from $100 \mathrm{eV}$ to $1000 \mathrm{eV}$. One expects to be able to detect interstellar matter in both absorption and emission. It is of critical importance to cosmology to determine the spectrum, isotropy and intensity of extragalactic radiation in this energy region. It is also expected that a large number of discrete sources with temperatures in the range from $10^{5} \mathrm{~K}$ to $10^{6} \mathrm{~K}$ will be detected. Such sources could result from neutron stars that are cooling or from the accretion of interstellar matter onto condensed objects. Matter falling into 'black holes' may be heated sufficiently to be detectable at long X-ray wavelengths. Extended objects, such as the Cygnus Loop and the Magellanic clouds, are known to be luminous at low X-ray energies (Grader et al., 1970; Gorenstein, 1970); other such objects are almost certainly present. There may be local sources of diffuse radiation. Finally, it should be possible to detect X-ray zodiacal light.

While a number of rocket studies of the low-energy X-ray sky have already been made, they all suffer from serious experimental problems and as yet our understanding of the long wavelength X-ray sky is very incomplete. There are sel ious disagreements between the various observers (Bowyer et al., 1968: Henry et al., 1968; Bunner et al., 1968: Baxter et al., 1969). The present apparatus was designed to overcome essentially all of the outstanding experimental problems. The instrument has been constructed, thoroughly tested, and successfully flown in an Aerobee-170 rocket. It clearly is a forerunner of a whole class of instruments of this type which will be needed to study the low-energy X-ray sky. An instrument somewhat similar to ours was recently flown by the AS\&E X-ray group in collaboration with our group.

The problems that previous experimenters faced all derived from the following facts: (1) Very thin counter windows are required to pass low-energy $\mathrm{X}$-rays and these windows are also transparent to precipitation electrons with energies of a few $\mathrm{keV}$; (2) the energy resolution of proportional counters is very poor at $\frac{1}{4} \mathrm{keV}$, being typically about $80 \%$, so that it is difficult to unequivocally separate high-energy $(1 \mathrm{keV})$ $\mathrm{X}$-ray events from low-energy $\left(\frac{1}{4} \mathrm{keV}\right)$ events; and (3) the thin window proportional counters have significant UV sensitivity and, in view of the poor pulse-height reso-

* This work was supported in part by the National Aeronautics and Space Administration under Grants NGR 33-008-102, NGR 33-008 012 and in part by the Air Force Office of Scientific Research under Grant AFOSR-70-1945. It is Columbia Astrophysics Laboratory Contribution No. 35. 
lution, it is difficult to separate single electron UV events from true X-ray events.

The present instrument utilizes a grazing incidence paraboloid as an energy concentrator and low-pass filter. The effective bandpass of the instrument from 100 to $280 \mathrm{eV}$ is determined by the combination of the counter window transmission and the mirror reflectivity. The instrument is provided with movable filters, one boron and the other Mylar. The boron filter substantially modifies the bandpass and the Mylar is used to determine the background since it is essentially opaque in the $100-280 \mathrm{eV}$ region. The reflection completely eliminates the problems arising from precipitation electrons and high-energy $X$ rays. The ultraviolet sensitivity of the instrument is minimized through careful design of the detector and the use of materials with high photoelectric work functions.

\section{Techniques}

Figure 1 illustrates the overall concept of the instrument. Photons are incident on a paraboloidal mirror and reflected to a proportional-counter detector. A collimator mask blocks all direct paths to the detector. The figure and coating of the mirror were selected to cut off all $X$ rays of energy greater than $0.35 \mathrm{keV}$. In addition to eliminating high-energy X-ray events, the mirror cuts off all (low-energy) charged particles. The detector is especially constructed to reduce sensitivity to UV.

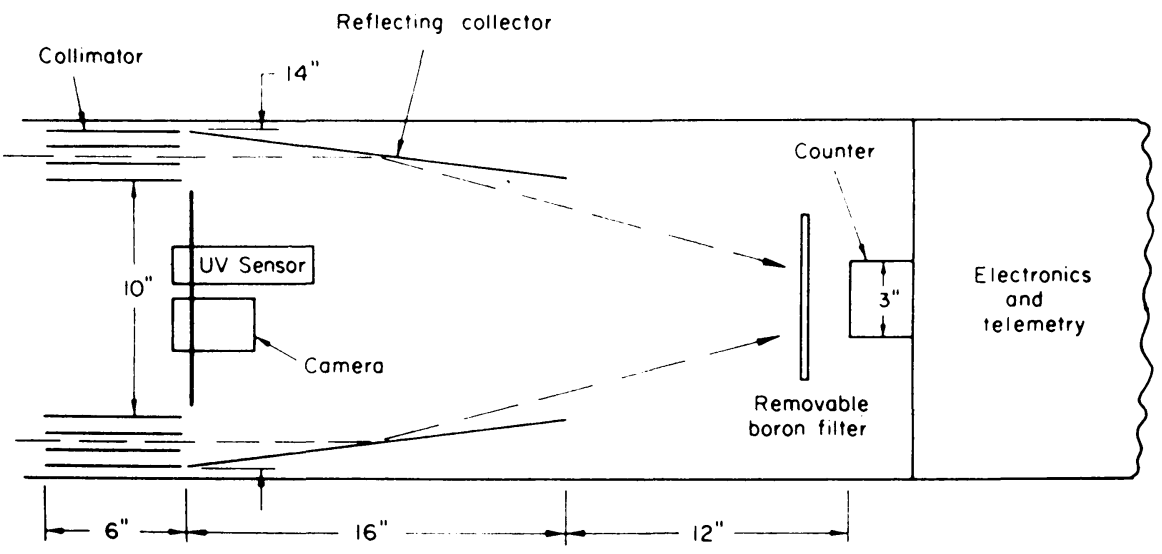

Fig. 1. Schematic diagram of the instrument.

The mirror provides two additional features besides energy filtering. One is an enhancement of the signal-to-noise ratio that results from a large collecting area and a small detector. The other is increased reliability, achieved by reducing the size of the window area. The very thin windows that must be used are fragile and this problem is clearly minimized by using small-area detectors mounted deep in the payload.

Since the present instrument was designed to eliminate high-energy photons and low-energy electrons, it employs only a single reflecting surface. This was done to 
achieve a large field of view and collecting area for the study of the low-energy diffuse X-ray background radiation. A single reflector of this type does not produce a true image of off-axis sources. It is clear that a true two-dimensional lens with double reflection must be used for a detailed mapping of the low-energy X-ray sky. Simple estimates indicate that the sensitivity of a rocket instrument of this type would be too small to provide a useful measure of the diffuse background, but it could be used to study discrete sources.

As indicated above, a boron filter was inserted on command in front of the counter to alter the spectral response. A Mylar filter was also available; it cuts off all reflected $\mathrm{X}$ rays and allows for an accurate measurement of the cosmic-ray-induced background without introducing a large amount of extraneous mass in front of the detector. The rocket aspect was recorded by a 35- $\mathrm{mm}$ camera and a 3-axis magnetometer mounted in the center of the collimator mask. Next to the camera was a photomultiplier sensitive to UV photons in the range 1500-2600 $\AA$. This detector was used to monitor the UV flux incident on the payload during flight, and was employed since we were concerned with the residual UV sensitivity of the instrument and it was important to have an independent direct measure of the incident $U V$ radiation.

The absolute sensitivity of the instrument to various discrete X-ray and UV lines was determined. For this purpose a fluorescence X-ray source provided photons at $0.18,0.26$, and $0.28 \mathrm{keV}$, (i.e., boron $\mathrm{K} \alpha$; potassium $\mathrm{L}$; carbon $\mathrm{K} \alpha$ ) and a number of higher energies. The fluorescent targets were excited with $\mathrm{Cu} \mathrm{L}$ lines from an X-ray tube constructed according to Henke (1967). A small bench vacuum chamber was used for detector window and filter calibrations; for calibrating the mirror response, a 44-m long vacuum chamber was used. This facility gave an X-ray beam parallel to

$4^{\circ}$. The payload was gimballed in a large chamber at the end of this beam, and the mirror response was determined for a number of orientations with respect to the beam.

\section{Details}

\section{A. MIRROR}

The surface of the mirror consists of the section from $z=31.6$ to $z=72.2$ of the paraboloid of revolution, $4.376 z=x^{2}+y^{2}$, where the dimensions are in centimeters. This surface has angles of incidence ranging from 7 to $10^{\circ}$ for axial rays. These steep angles were chosen to cut off all $\mathrm{X}$ rays except those with energy less than $0.35 \mathrm{keV}$ and to maximize the area of the collector. The mirrors were constructed by blowing Pyrex (Corning 7740) glass into a conical mold followed by fine annealing. The parabolic surface was ground and polished by standard techniques. The figure was held only crudely, the requirement being a focal spot of $\frac{1}{2}$ in. diameter for axial rays. However, the polishing was carefully done to give the smoothest possible surface. The mirror surface was checked by replica electron microscopic studies of sample areas. These studies and the overall efficiency measurements indicated that the mirror polish was sufficient to insure that the reflectivity was essentially the same as that measured on high quality optical flats. It should be emphasized that we were not attempting to 
achieve a high resolution image and there may be appreciable small angle scattering of the $\mathrm{X}$ rays.

The mirrors were coated with approximately $1000 \AA$ of chromium. This greatly enhanced the reflectivity for soft $X$ rays at large angles of incidence. The reflectivity of optical quality glass flats was measured with a variety of coatings at a number of X-ray energies. The results, illustrated in Figure 2, show $\mathrm{Cr}$ to be the best coating in agreement with previous measurements (Lukirskii et al., 1964). It provides not only the largest reflectivity at the largest angles for the energy range $E<0.28 \mathrm{keV}$, but can be repeatedly cleaned and shows no signs of deterioration with age. For example, there was no perceptible decrease in reflectivity in both the test flats and the paraboloid after several months' storage in New York City air. Furthermore, a mirror with a contaminated surface was cleaned with standard nonabrasive laboratory detergents and restored to its original efficiency.

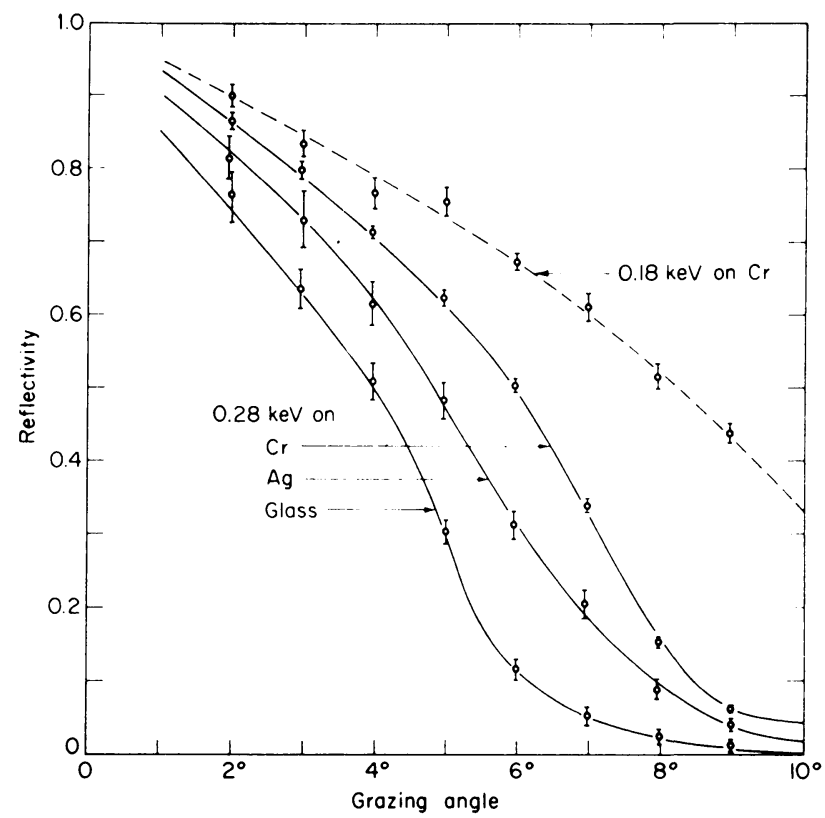

Fig. 2. Reflectivity of glass and silver for $280 \mathrm{eV}$ photons, and chromium for $180 \mathrm{eV}$ and $280 \mathrm{eV}$ photons.

The glass mirrors were mounted by suspending them in an oversized metal cone and filling the gap between the glass and the cone with silicone-rubber potting compound loaded with small hollow phenolic spheres to reduce density and increase resiliency. This mounting technique enabled the mirror to easily survive all environmental tests and the flight in an Aerobee-170 rocket.

The mirrors were calibrated in monochromatic, parallel beams of soft $\mathrm{X}$ rays available in the vacuum facility mentioned above. The beam intensity was measured, 
using the payload detector, by covering the annular aperture of the mirror and opening the central area of the collimator mask (see Figure 1). The central area was then blocked and the annular aperture opened to obtain the mirror efficiency. Figure 3 shows the measured effective area of the mirror for three X-ray energies as a function of angle between the X-ray beam and the axis of the mirror. For comparison, the projected geometrical area of the lens is $550 \mathrm{~cm}^{2}$.

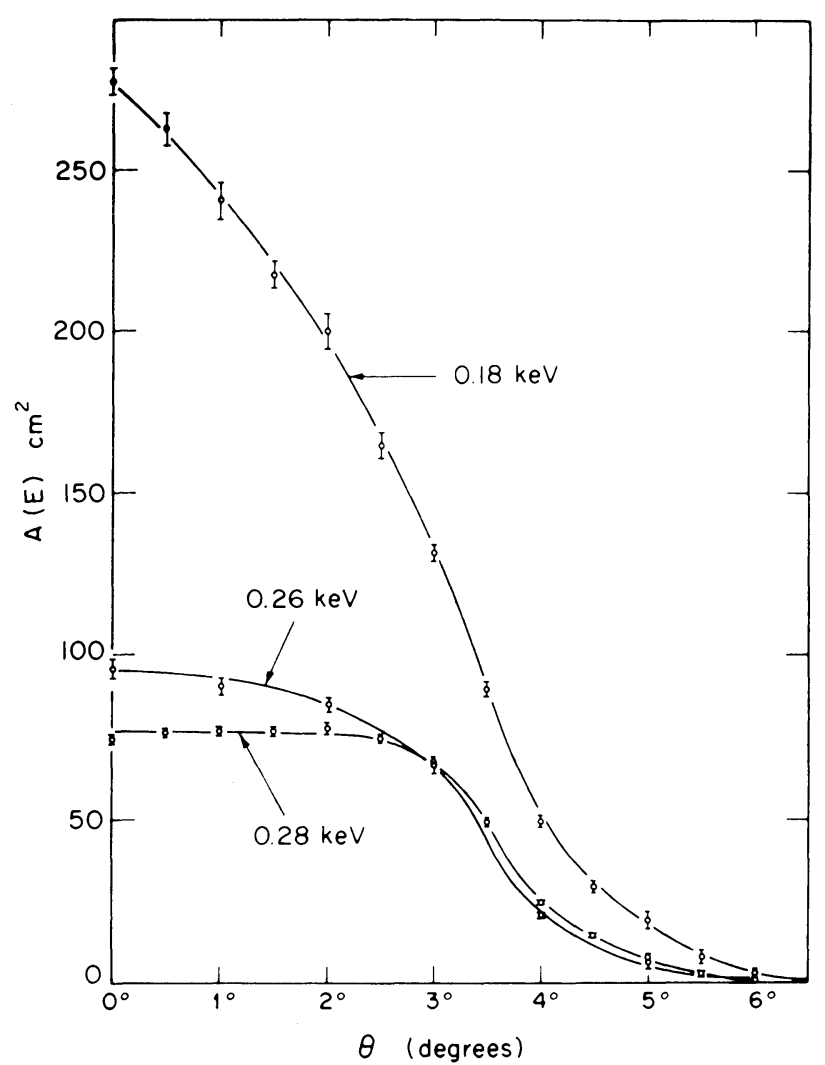

Fig. 3. Effective area of the mirror-collector for 180,260 and $280 \mathrm{eV}$ X-rays as a function of angle between the X-ray beam and the mirror axis.

\section{B. THE DETECTOR}

The detector is located at the focus of the mirror and is shown in Figure 4. It consists of a large volume $(5 \mathrm{~cm} \times 15 \mathrm{~cm} \times 10 \mathrm{~cm})$ of pure methane counting gas at 1 atm pressure, which is divided by thin $(0.08 \mathrm{~mm})$ ground wires into seven individual counters. The three counters just behind the window are the signal counters and the four surrounding counters provide anticoincidence shielding. An $\mathrm{Fe}^{55}$ monitor counter shares the gas and the high voltage of the signal counters but is located in a separate chamber. The pulse-height spectrum from this counter was used to actively control the gain of the system. The anode wires were tungsten $0.025 \mathrm{~mm}$ in diameter except the $\mathrm{Fe}^{55}$ mo- 


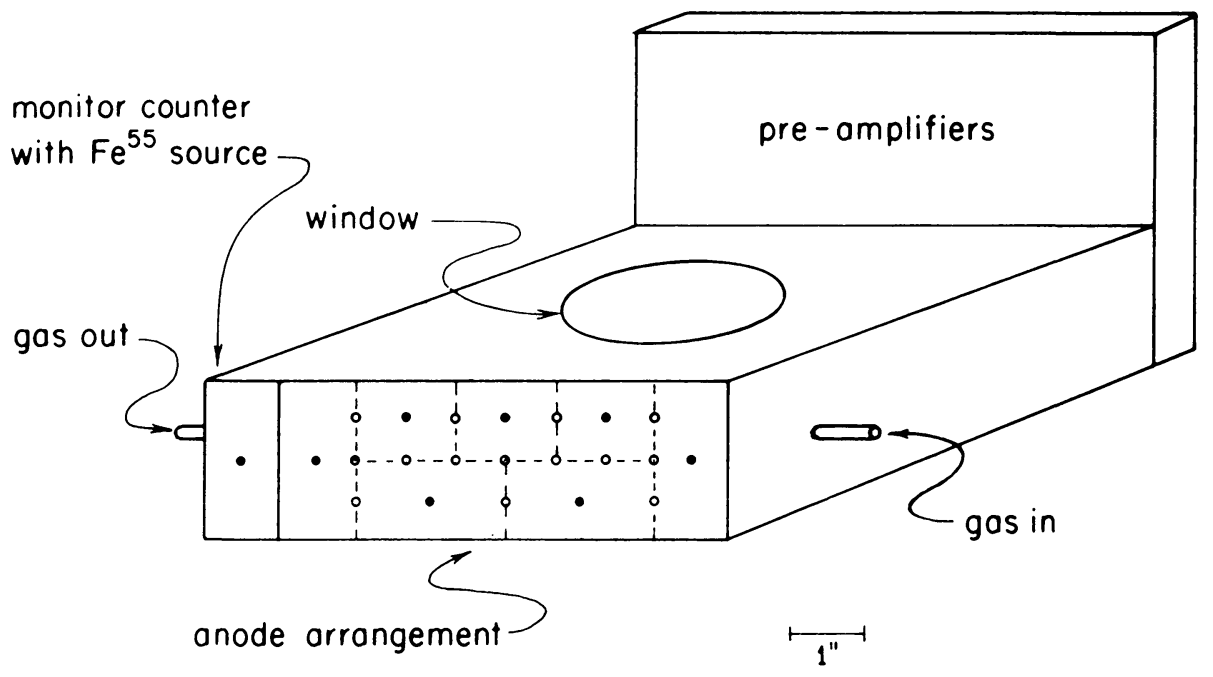

Fig. 4. Diagram of the detector.

nitor counter which had an anode $0.051 \mathrm{~mm}$ in diameter. This causes the $5.9 \mathrm{keV}$ iron line in the monitor counter to appear at the same pulse height as that of an approximately $\frac{1}{4} \mathrm{keV}$ line in the signal counters. Separating the signal and anticoincidence counters by only a few thin wires rather than a solid wall allows better rejection of the background events induced by cosmic rays because a substantial fraction of these events are caused by Compton electrons produced in the wall material. Furthermore, the UV sensitivity is reduced by this design. Long wavelength UV produces photoelectrons in the walls rather than in the gas. With this construction these electrons are released in the anti-shield counter, not in the signal counters. Thus the only source of UV photoelectrons and Compton electrons in the signal counter is the front window and the anode and ground wires.

To further reduce UV sensitivity, the counter walls were coated by spraying them with a suspension of colloidal graphite in alcohol. T his reduces the photoelectric yield by a factor of 10-100 over bare aluminum. The window was similarly coated with $75 \mu \mathrm{g} / \mathrm{cm}^{2}$ of graphite rather than aluminum to provide a conductive coating. Aluminum is particularly poor as a coating for counter windows because although the UV photons are stopped by the coating, photoelectrons from the absorption process may be injected directly into the counter.

The window consisted of $1 \mu$ polypropylene, $\left(\mathrm{CH}_{2}\right)_{n}$, produced by stretching an unoriented $0.0254-\mathrm{mm}$ polypropylene sheet to this thickness. The plastic was mounted on a coarse mesh of stainless steel screen with a geometrical transmission of $61 \%$. The windows were $7.6 \mathrm{~cm}$ in diameter, this diameter corresponding to the $6^{\circ}$ diameter field of view of the mirror. The windows withstood a large number of vacuum cycles without leaking.

Calibration of the windows was achieved by inserting them into a monochromatic 
beam and recording the attenuation produced. Figure 5 shows the transmission for a typical window at several energies; losses due to the mounting mesh are not included. The line is a theoretical fit to the calibration data using tabulated mass attenuation coefficients (Henke, 1967). Figure 6 gives the overall response of the system to diffuse

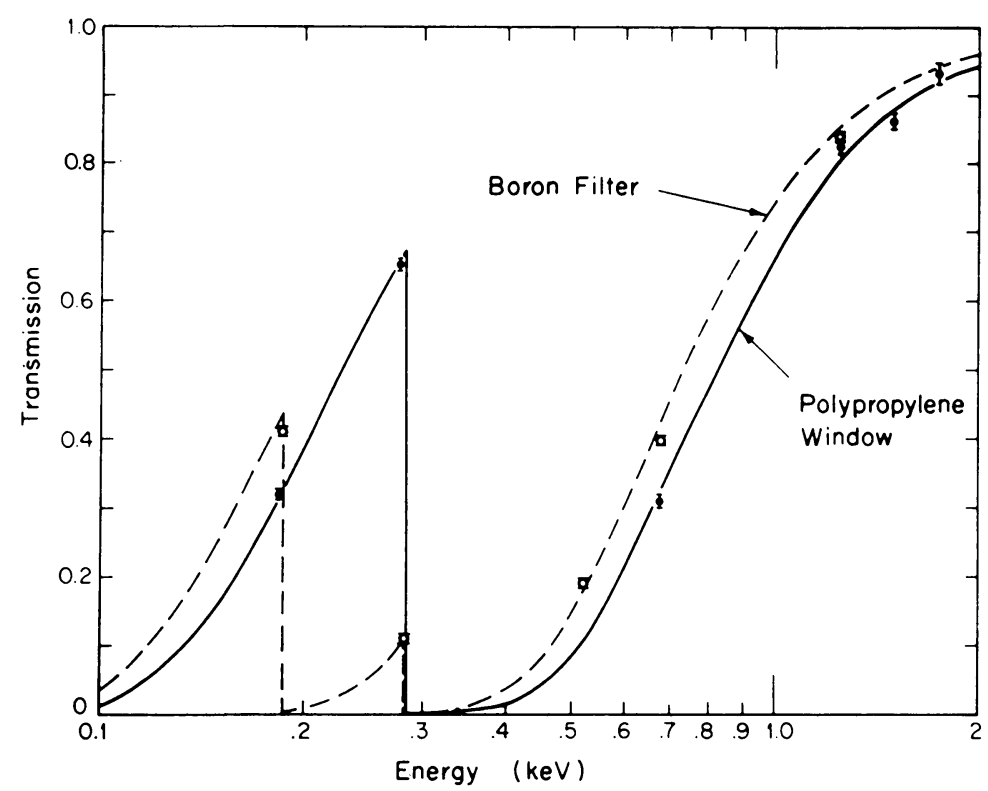

Fig. 5. Transmission of the polypropylene window and the boron filter as a function of photon energy.

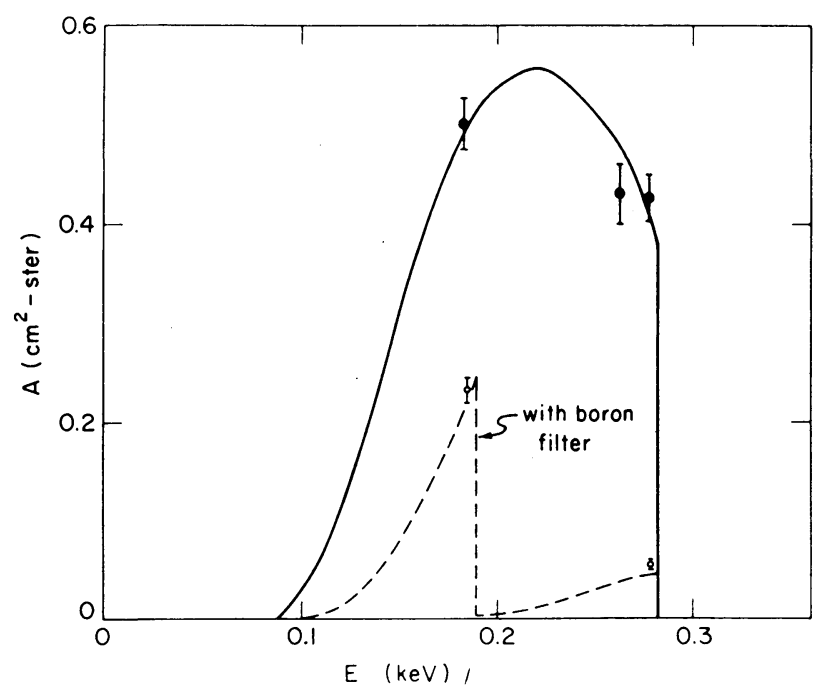

Fig. 6. Overall response of the system to diffuse soft X-rays. 
X-rays. The energy response of the instrument is well established, not only in the desired X-ray band but also in the UV region.

The counter for the $\mathrm{Fe}^{55}$ line monitor was identical to the signal counters. Since the servo circuitry required a counting rate of $3000 \mathrm{cps}$ and since the counter is quite inefficient at $6 \mathrm{keV}(0.1 \%)$, it was necessary to use a 100 -microcuries source of $\mathrm{Fe}^{55}$. Operation for extended periods of time at these rates degrades the counter resolution. This is presumably due to deposits building up on the anode wire; replacing the wire restores the resolution. The use of stainless steel anode wire rather than tungsten has been suggested to minimize this effect. Organic gases such as methane are known to be particularly bad in this respect, but methane was used because it provides highenergy resolution at low X-ray energies. A gas flow system is used because of the semi-porous nature of the counter window. The gas system consisted of two $\frac{1}{2}$ liter storage tanks (800 psi), an Airco miniature absolute pressure regulator, pressure monitors, and a sintered, porous-plug leak on the gas exit of the counter which allowed a flow into a vacuum of a few cubic centimeters per second. All of the calibrations were performed under the same flow conditions as those experienced in flight. A photograph of the mirror-detector configuration is shown in Figure 7.

\section{ELECTRONICS}

Figure 8 shows a block diagram of the electronics. Signals from the anodes of the various counters were processed through identical charge-sensitive preamplifiers,

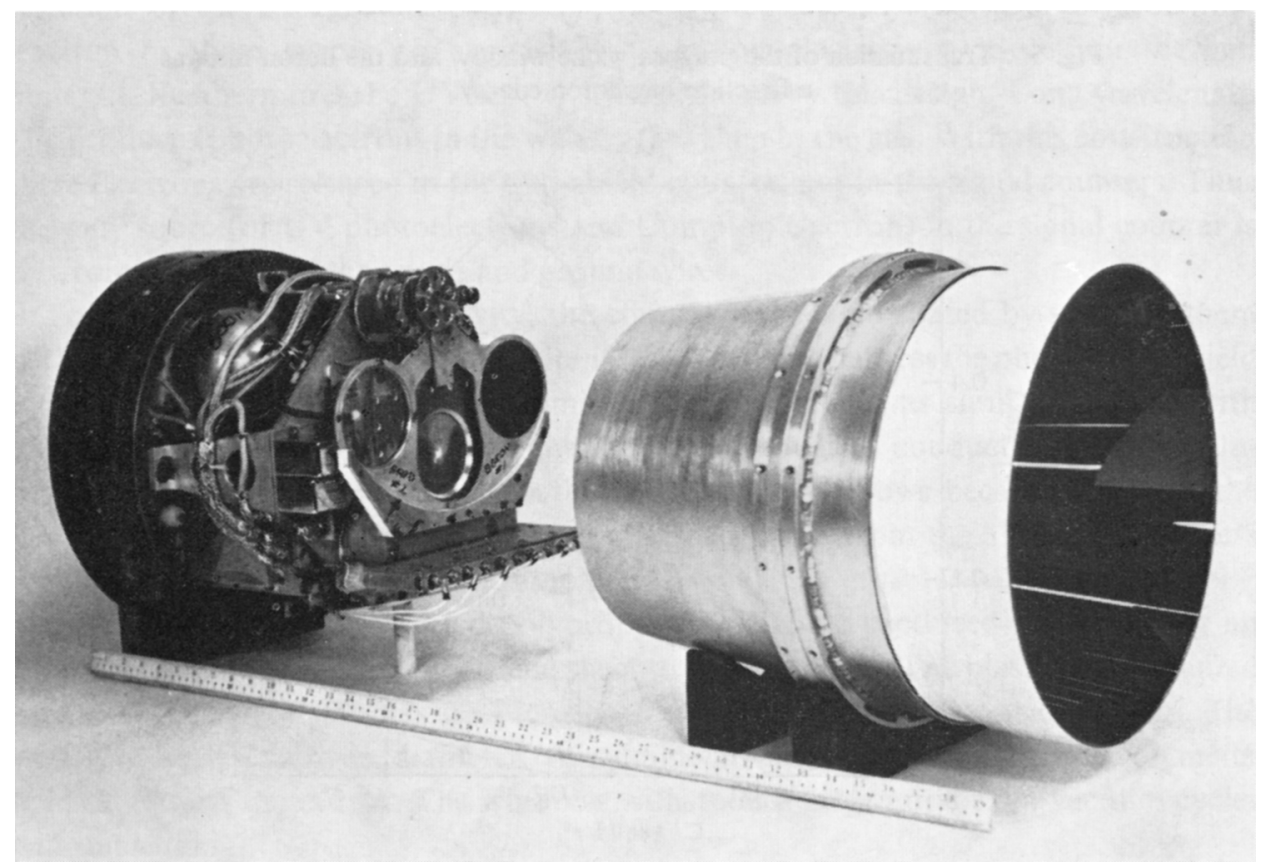

Fig. 7. The mirror-detector-filter-gas supply system. 
discriminators, and pulse stretchers before presentation to the $20-\mathrm{kHz}$ PPM-AM telemetry system. The discriminators were set to trigger at $25 \mathrm{mV}$, about six to eight times the noise level of the preamplifiers. Upon triggering, the pulses were stretched to $2 \mathrm{mS}$ width with the height preserved. In addition, the signal channels contained logic circuits which vetoed events occurring in coincidence with the antichannel. The minimum output level to the telemetry was $0.5 \mathrm{~V}$, corresponding to $100 \mathrm{eV}$ deposited in the counter.

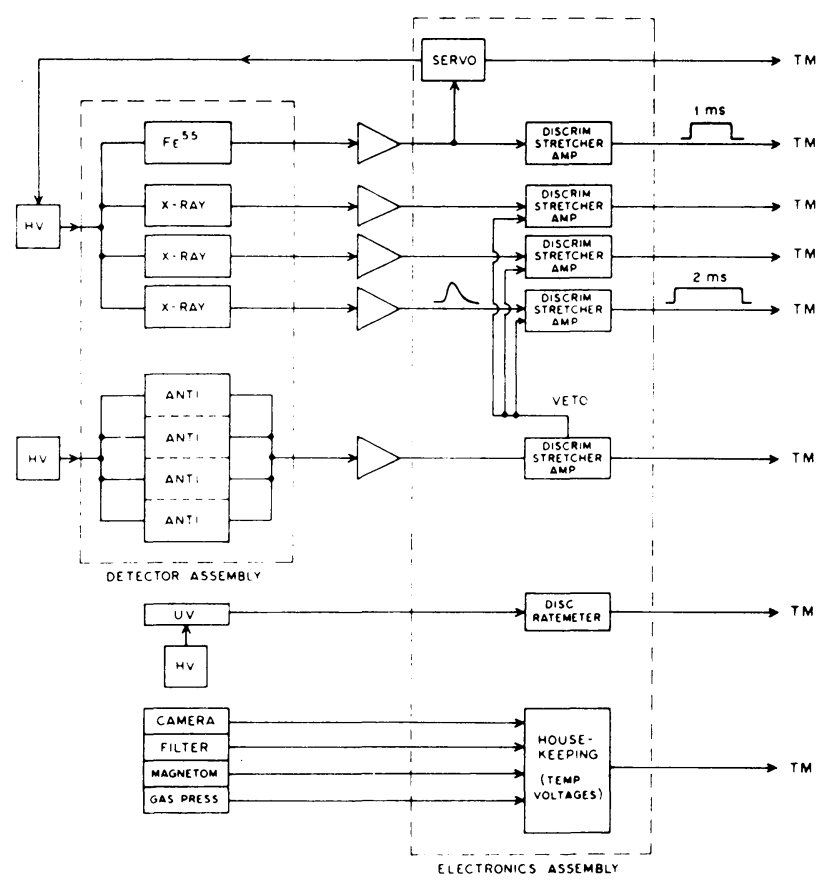

Fig. 8. Block diagram of the electronics system.

The gain and resolution of the counter-electronics system was checked by recording pulse-height spectra of monochromatic X rays. Figure 9 shows two such spectra, one from carbon $\mathrm{K} \alpha$ at $0.28 \mathrm{keV}$, the other from boron $\mathrm{K} \alpha$ at $0.18 \mathrm{keV}$. The resolution is $70 \%$ and $90 \%$, respectively, for these two lines.

The servo circuit controlled the gain of the system by comparing the number of events in two adjacent pulse-height windows. If the $\mathrm{Fe}^{55}$ line was split equally into these windows, the high voltage $(\simeq 3000 \mathrm{~V})$ was correct. An error signal proportional to the difference in the counting rate between the windows was used to correct the output of an externally programmable high voltage supply. Several safety features were incorporated into the control circuit, the most important being a search mode which caused the servo to hunt for the correct high voltage if the servo ever lost control.

The electronics package also included all of the power supplies, voltage monitors, temperature monitors, and function monitors that make up the housekeeping of a 
rocket payload. One of these monitors, the UV monitor, had a continuous data channel. Counts from the UV photomultiplier were detected by a discriminator trigger circuit and used to charge a capacitor. The output of this capacitor ratemeter was a ramp which was reset every 900 counts.

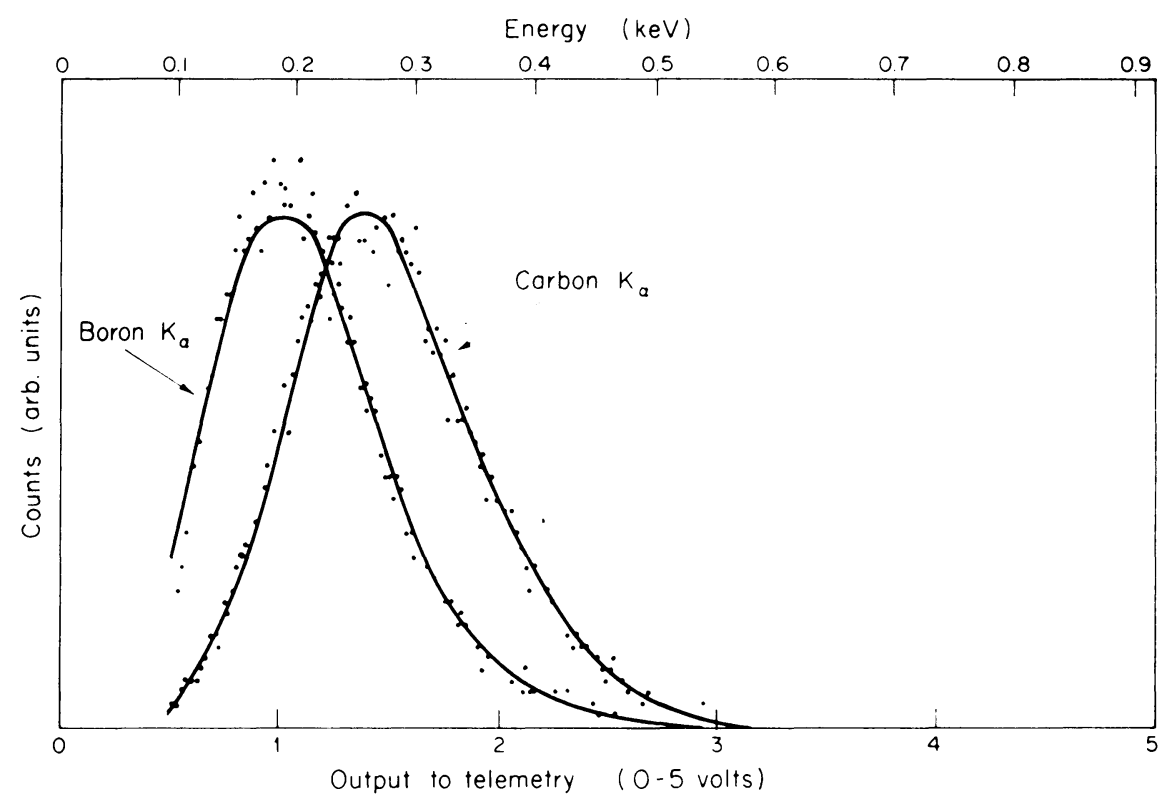

Fig. 9. Pulse-height spectra of carbon $\mathrm{K} \times$ and boron $\mathrm{K} \sim$ radiation.

\section{FILTERS}

The boron and the Mylar filters were mounted in a mechanism which drove them into and out of place on command from the rocket ACS system. The Mylar filter consisted simply of $0.25-\mathrm{mm}$ Mylar bonded to a thin ring. It stopped all low-energy $\mathrm{X}$ rays (high-energy $X$ rays being cut off by the mirror), and was used at the beginning and end of the flight to measure the background counting rate induced by cosmic rays.

The boron filter was constructed by bonding 1- $\mu$ polypropylene to a thin ring and spraying the polypropylene with a suspension of approximately $100-\AA$ diameter boron particles in alcohol to an effective thickness of $55 \mu \mathrm{g} / \mathrm{cm}^{2}$ of boron. The filter was calibrated for X-ray transmission by the technique used for the windows, and the dotted line in Figure 4 gives the transmission of a typical filter. The shift in spectral response on inserting this filter provides spectral information on the incident spectrum. This information is difficult to extract reliably from the pulse-height spectrum alone because of the broad counter resolution.

\section{E. UV MONITOR AND CAMERA}

The UV monitor consisted of an EMR photomultiplier mounted to have a field of view of 0.04 ster along the rocket axis. The phototube was sensitive from 1800 to 


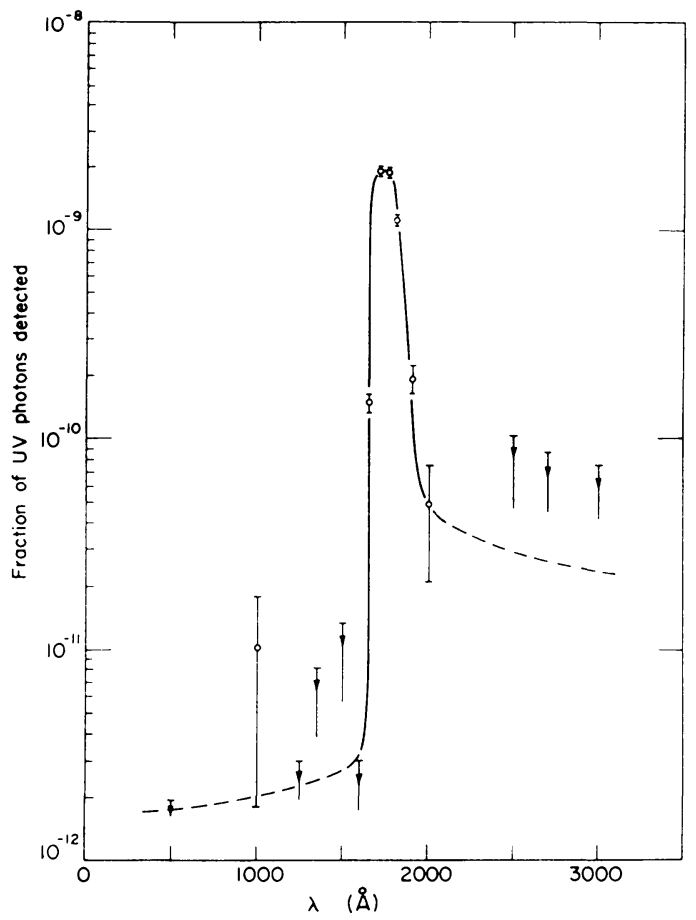

Fig. 10. UV quantum efficiency of the proportional counter with the lower pulse-height level at $0.5 \mathrm{~V}$ as in flight.

$2600 \AA$ and was calibrated on a McPherson UV monochromator. The integrated response, that is, the product of the area, solid angle factor and quantum efficiency over the spectral range is $3.0 \times 10^{-3} \mathrm{~cm}^{2}$ ster $\AA$. Hydrogen Lyman- $\alpha$ was not monitored because the signal would have been so large as to mask the radiation that was potentially more dangerous. Lyman- $\alpha$ is stopped very effectively by the proportional counter plastic window, but longer wavelengths are not stopped so completely. The detector was also calibrated for UV sensitivity (see Figure 10). The integrated response of the system from $500-3000 \AA$ is $2 \times 10^{-7} \mathrm{~cm}^{2}$ ster $\AA$, assuming the mirror to be perfectly reflecting. The reason for the relatively large response at $1700 \AA$ is not understood.

The aspect camera took about thirty 5-s exposures during the flight. It was built by the space science group at Kitt Peak National Observatory. Stars as faint as 5th mag were recorded on exposures made when the rocket was not scanning. The aspect could be determined to $0.1^{\circ}$; the errors arise largely from uncertainties in alignment of the payload components.

\section{Instrument Performance and Conclusions}

The instrument was flown on February 14,1970 at $05^{\mathrm{h}} 30^{\mathrm{m}} 01^{\mathrm{s}}$ UT in an Aerobee-170 rocket launched from the White Sands Missile Range. The instrument survived flight 
without damage, the counter window and filters did not tear, and the high voltage servo on the proportional counter remained locked during lift-off and the entire flight. The flight was programmed so that the instrument scanned from the galactic plane to a point near the north galactic pole. The Mylar filter was inserted for a short time early in the flight and again late in the flight for background determination. The boron filter was inserted to obtain spectral information in the vicinity of the galactic pole. The complete analysis of the flight data will be published elsewhere. Here, we will only discuss the performance of the instrument as evidenced by the counting rate and pulse-height spectra. From the preflight calibration data (Figures 6 and 9), $100-280 \mathrm{eV}$ X-ray events correspond to $0.5-3.0 \mathrm{~V}$ pulses presented to the telemetry system. The counting rate in this pulse-height interval is shown in Figure 11.

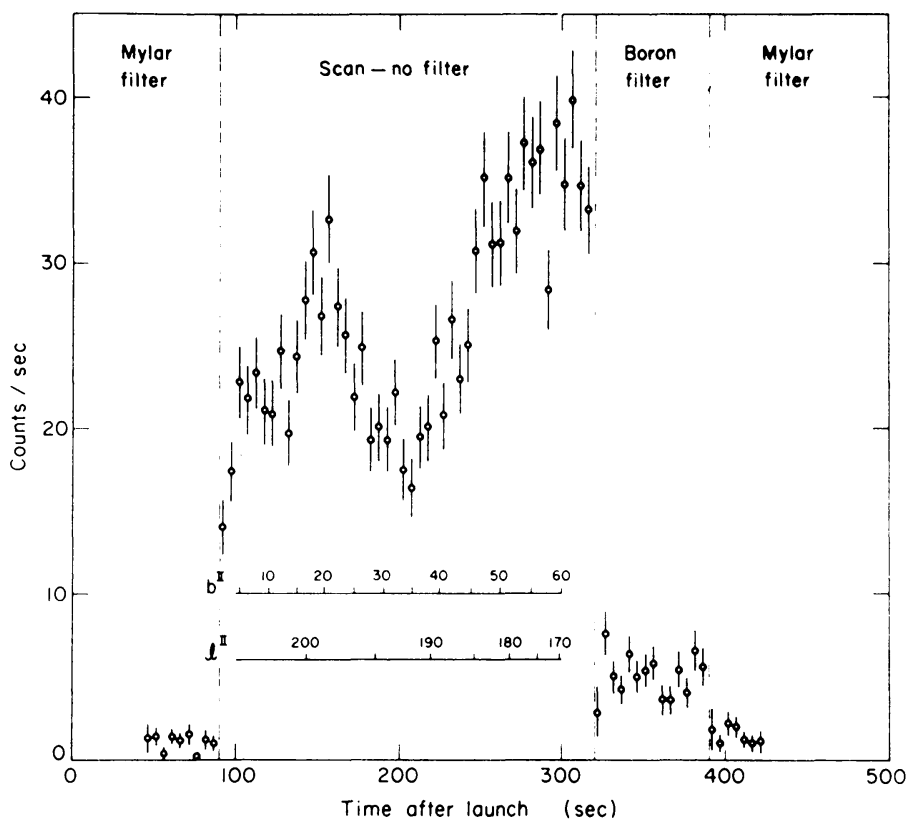

Fig. 11. Counting rate during flight $-5 \mathrm{~s}$ averages.

The background pulse-height spectrum obtained with the Mylar filter is shown in Figure 13. Here we note that the spectrum is approximately flat from about $2.0 \mathrm{~V}$ to the overflow bin at $5.0 \mathrm{~V}$ and that there is a gradual increase in the pulse-height spectrum below $2.0 \mathrm{~V}$. This trend is similar to that obtained by other observers. The total background counting rate in the three signal counters in the pulse-height range from 0.5 to $3.0 \mathrm{~V}$ is 1.3 counts per second. In arriving at this result, we have excluded those events which occurred simultaneously in two or more signal counters. Without this final anticoincidence veto, the background rate would have been 3.6 counts per second. Taking the area of the signal counters to be $100 \mathrm{~cm}^{2}$, the background flux is 0.013 counts $/ \mathrm{cm}^{2} \mathrm{~s}$. Taking the effective energy of the background events as $200 \mathrm{eV}$ 


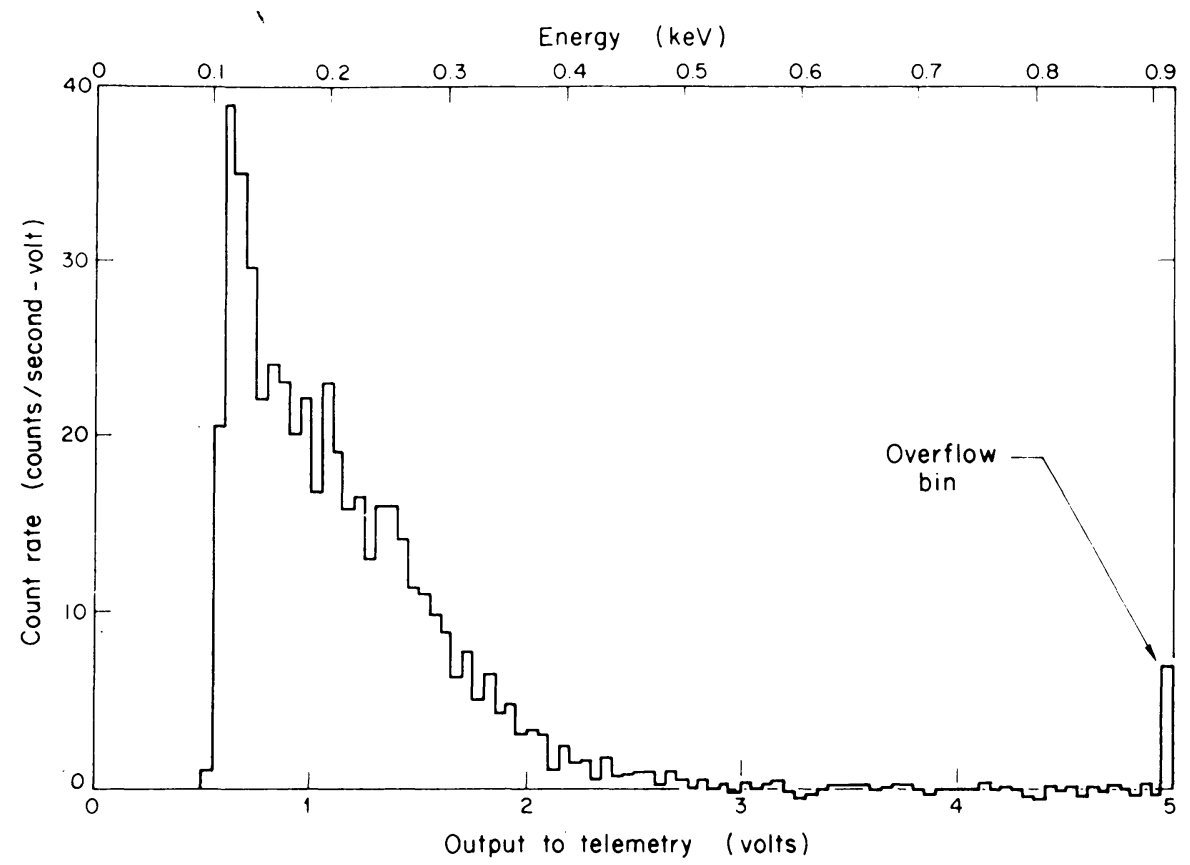

Fig. 12. Pulse-height distribution (data during scan).

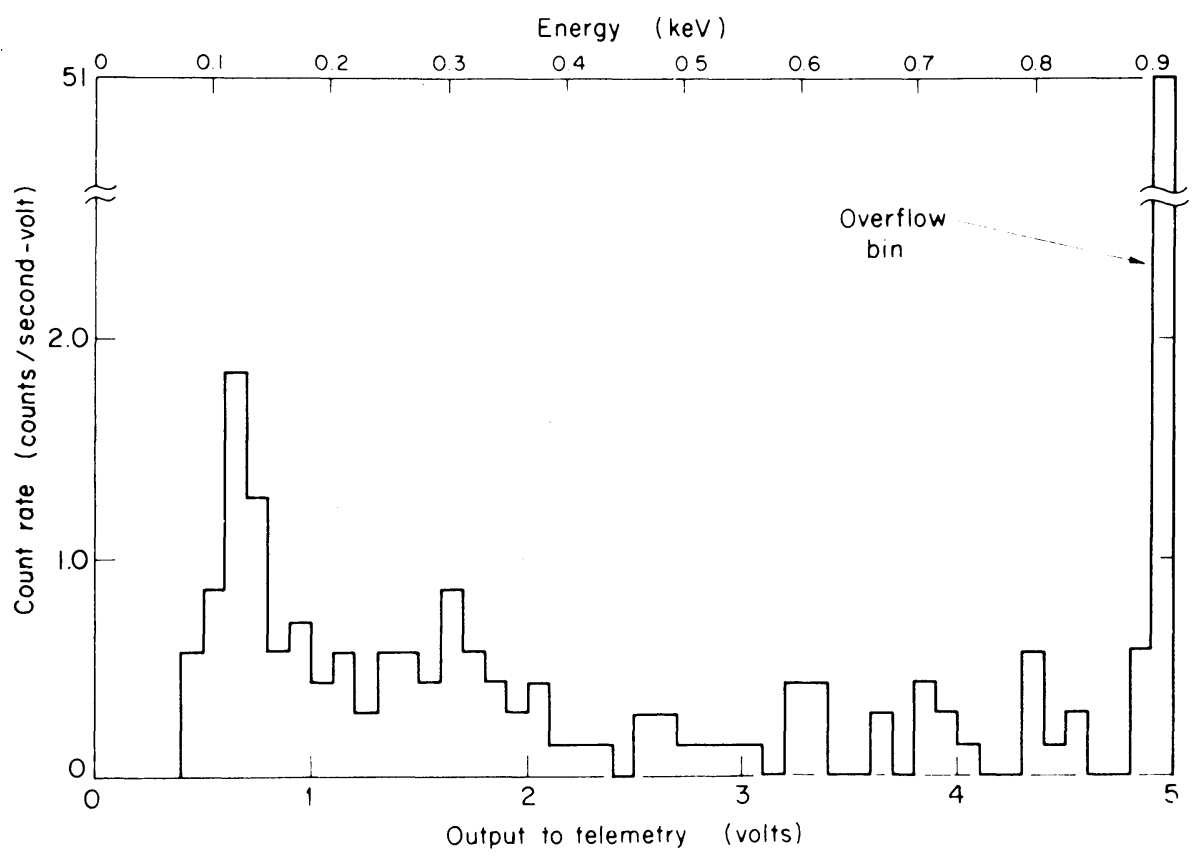

Fig. 13. Background pulse-height distribution (with Mylar filter). 
and the energy bandwidth as $300 \mathrm{eV}$ for events in 0.5 to $3.0 \mathrm{~V}$ pulse-height range, the apparent average spectral background flux is $0.008 \mathrm{keV} / \mathrm{keV} \mathrm{cm} \mathrm{c}^{2} \mathrm{~s}$. Possibly by employing pulse-shape discrimination and other background rejection techniques, it may be possible to reduce this value to that obtained at higher energies; i.e., 0.001 $\mathrm{keV} / \mathrm{keV} \mathrm{cm}^{2} \mathrm{~s}$.

It is noteworthy that the flight data contained no signals larger than those expected from $280 \mathrm{eV}$ photons (Figure 12) showing that, in fact, all high-energy X-rays and electrons were eliminated by the lens. It is somewhat more difficult to determine if we were equally successful in eliminating UV contamination. There are some data late in the flight when the instrument was re-entering the atmosphere that suggest that some of the counts close to the lower level threshold were due to UV radiation. This point will be discussed in more detail in our paper on the analysis of the results.

The results of the flight strongly indicate that the instrument performed as expected and that we completely eliminated two important sources of systematic error present in previous studies of low-energy celestial X-rays. Clearly, larger instruments and longer observing times are necessary for the full mapping of the low-energy X-ray sky. In addition, high-resolution focusing instruments will be required to eliminate source confusion. The techniques presented here are clearly essential to the success of future long wavelength X-ray astronomy.

\section{References}

Baxter, A. J., Wilson, B. G., and Green, D. W.: 1969, Astrophys. J, 155, L145.

Bowyer, C. S., Field, G. B., and Mack, J. E.: 1968, Nature 217, 32.

Bunner, A. W., Coleman, P. C., Kraushaar, W. L., McCammon, D., Palmieri, T. M., Shilipelay, A., and Ulmer, M.: 1968, Nature 223, 1222.

Gorenstein, P.: 1970 (private communication).

Grader, R. J., Hill, R. W., and Stoering, J. P.: 1970 (preprint).

Henke, B. L.: 1967 Norelco Reporter XIV,75.

Henry, B. C., Fritz, G., Meekins, J. F., Friedman, H., and Byram, E. T.: 1968, Astrophys. J. 153, L11.

Lukirskii, A. P., Savinov, E. P., Ershov, O. A., and Shepelev, Yu. F.: 1964, Opt. Spectr. 16, 168. 